\title{
Effect of radiotherapy on brain glucose metabolism in patients operated on for low grade astrocytoma
}

\author{
M Bruehlmeier, U Roelcke, B Amsler, K H Schubert, O Hausmann, K von Ammon, \\ E W Radü, O Gratzl, C Landmann, K L Leenders
}

\begin{abstract}
Objective-To assess the effect of postoperative radiotherapy on brain glucose metabolism (CMRGlu) of operated patients with low grade astrocytomas. Methods-PET and ${ }^{18}$ F-fluorodeoxyglucose was used to measure absolute CMRGlu in patients with fibrillary astrocytoma (WHO II) of the frontal lobe, who did $(n=7)$ or did not $(n=12)$ receive radiotherapy subsequent to first debulking tumour resection. In addition, statistical parametric mapping (SPM95) was applied to assess the pattern of relative CMRGlu associated with the frontal tumour. Data were compared with 12 healthy controls.

Results-A global reduction of absolute CMRGlu was found when either patients with or without radiotherapy were compared with controls (ROI analysis). Brain areas of relative CMRGlu reduction were found in the brain ipsilateral and contralateral to the tumour, comparing both patient groups with controls by SPM ("tumour diaschisis effect"). Superimposed, absolute CMRGlu in the contralateral frontal, parietal, occipital cortex as well as in the white matter was on average $17 \%$ lower in patients receiving radiotherapy than in patients who did not.

Conclusions-The data discriminate a tumour effect from a radiotherapy effect, and support the view of adverse effects of radiotherapy on brain not directly involved by tumour.
\end{abstract}

(F Neurol Neurosurg Psychiatry 1999;66:648-653)

Keywords: low grade astrocytoma; brain glucose metabolism; radiotherapy

Low grade astrocytomas (LGAs) account for 30 to $40 \%$ of all gliomas. After surgery, patients with LGA may live for several years relatively symptom free with an average interval of 2 to 4 years until tumour recurrence. A considerable number of patients deteriorate more rapidly due to tumour progression towards higher malignancy, which may drastically reduce the quality and duration of life. Up to $50 \%$ of
LGAs recur as malignant tumours at a mean postoperative interval of 31 to 56 months. ${ }^{1}$

Whereas most authors agree that the extent of tumour resection correlates with the survival time of patients with LGAs, ${ }^{2}$ there is a controversial debate about the benefit and side effects of early postoperative radiotherapy. ${ }^{13-6}$ The principal idea of radiotherapy is to destroy anaplastic cell foci, which may be already present in LGAs. However, most recommendations of the use of radiotherapy are based on retrospective clinical data, which in part are hampered by heterogeneous patient samples. One prospective study comparing the effect of different radiation doses failed to establish a correlation between dose and survival of patients with LGAs.

In most cases, radiotherapy of patients with brain tumours is associated with irradiation of tumour adjacent or remote brain, and side effects of radiotherapy have to be considered carefully. Several authors have proposed that cognitive impairment is a major complication of radiotherapy, but this has been questioned by others (for review see Roman and Sperduto ${ }^{8}$ ). In the present study we set out to investigate the effect of external radiotherapy from a metabolic point of view. We used PET and the tracer ${ }^{18} \mathrm{~F}$-fluorodeoxyglucose (FDG) to investigate cerebral (non-tumorous) glucose metabolism, which can be used as a measure of neuronal activity, ${ }^{9}$ in patients with LGAs who did or did not receive external radiotherapy subsequent to their first debulking tumour resection. Particular attention was given to separating the effect of residual or recurrent tumour from the effect of radiotherapy on cerebral glucose metabolism (CMRGu).

\section{Patients and methods}

Depending on location and size, circumscribed brain lesions may suppress remote CMRGu and blood flow (diaschisis ${ }^{10}$ ). To minimise the diaschisis effect which could arise from various tumour locations, we included only tumours which were confined to the frontal lobe of one brain side (according to MRI). In addition, only tumours which at the time of the PET study did not show MRI criteria of malignancy (contrast enhancement, peritumorous oedema) were and in revised form

6 August 1998

Accepted 29 October 1998 
Table 1 Clinical data

\begin{tabular}{|c|c|c|c|c|c|c|}
\hline & Age & Sex & $\begin{array}{l}\text { Resection } \\
\text { type }\end{array}$ & $\begin{array}{l}\text { Interval } \\
\text { (months) }\end{array}$ & $\begin{array}{l}\text { Tumour size } \\
\left(\mathrm{mm}^{2}\right)\end{array}$ & CMRGIu \\
\hline \multirow[t]{7}{*}{ RADY } & 34 & $M$ & $\mathrm{P}$ & 25 & 314 & $\mathrm{~N}$ \\
\hline & 72 & $\mathrm{~F}$ & $\mathrm{P}$ & 22 & 225 & $\mathrm{~N}$ \\
\hline & 36 & M & GT & 17 & 174 & $\mathrm{~N}$ \\
\hline & 25 & $M$ & $\mathrm{P}$ & 28 & 440 & $\mathrm{Y}$ \\
\hline & 39 & $\mathrm{~F}$ & $\mathrm{P}$ & 24 & 226 & $\mathrm{Y}$ \\
\hline & 45 & M & $\mathrm{P}$ & 14 & 121 & $\mathrm{Y}$ \\
\hline & 37 & $\mathrm{~F}$ & $\mathrm{P}$ & 42 & 660 & $\mathrm{Y}$ \\
\hline Mean (SD) & $41(15)$ & & & $46(32)$ & 308 (186) & \\
\hline \multirow{12}{*}{ RADN } & 44 & M & GT & 7 & 438 & $\mathrm{Y}$ \\
\hline & 34 & $\mathrm{~F}$ & $\mathrm{P}$ & 27 & 530 & $\mathrm{Y}$ \\
\hline & 48 & $\mathrm{~F}$ & $\mathrm{P}$ & 58 & 200 & $\mathrm{Y}$ \\
\hline & 39 & $\mathrm{~F}$ & GT & 120 & 283 & $\mathrm{~N}$ \\
\hline & 40 & $\mathrm{~F}$ & GT & 15 & 729 & $\mathrm{~N}$ \\
\hline & 36 & $\mathrm{~F}$ & GT & 51 & 200 & $\mathrm{~N}$ \\
\hline & 51 & M & $\mathrm{P}$ & 64 & 550 & $\mathrm{~N}$ \\
\hline & 65 & $M$ & $\mathrm{P}$ & 84 & 537 & $\mathrm{~N}$ \\
\hline & 30 & M & $\mathrm{P}$ & 21 & 207 & $\mathrm{Y}$ \\
\hline & 27 & $M$ & $\mathrm{P}$ & 23 & 748 & $\mathrm{Y}$ \\
\hline & 52 & $\mathrm{~F}$ & $\mathrm{P}$ & 81 & 298 & $\mathrm{Y}$ \\
\hline & 29 & M & $\mathrm{P}$ & 30 & 231 & $\mathrm{Y}$ \\
\hline Mean (SD) & $41(11)$ & & & $48(34)$ & $413(204)$ & \\
\hline
\end{tabular}

RADN=non-irradiated patients; RADY=irradiated patients; GT=gross total (macroscopically complete); $\mathrm{P}=$ partial resection. Interval=interval between tumour resection, (with or without radiotherapy) and PET study Tumour size was assumed to correspond to the area calculated from the axial plane (CCT or MRI, available as films) which showed the greatest diameter of resident/ recurrent tumour. From that plane, the largest diameter was determined (x). Vertical to that line, a second diameter was determined (y). Tumour size was then calculated as elliptical area as: $\pi \times \mathrm{x} /$ $2 \times y / 2$. For CMRGlu Y=arterial blood samples available.

included. Applying these criteria, we retrospectively analysed FDG PET images of 19 (seven irradiated, 12 non-irradiated) patients who at the first operation presented with fibrillary astrocytoma of the frontal lobe (WHO II). Both patient groups were controlled for age, interval from operation, with or without radiotherapy, to PET study, resection type, and tumour size. At the time of the PET study, no patient received dexamethasone. No patient was previously treated with chemotherapy. The clinical details are presented in table 1 . For comparison, data from 12 healthy subjects were included (mean (SD) age 39 (13), range 24 to 64 years, three women, nine men). The permission to perform FDG PET studies in patients and controls was provided by the ethics committee of the University Hospitals Zürich and Basel.

RADIOTHERAPY

The decision to treat patients with radiotherapy did not depend on clinical or neuroradiological criteria, but was defined by the policy of the referring clinical department. Whereas patients from Zürich were not irradiated, all patients from Basel received radiotherapy. Patients were immobilised with a thermoplastic head fixation to ensure reproducible position. CT based dose planning was done on a Philips TPS. The preoperative tumour volume with a $2 \mathrm{~cm}$ margin was chosen as the planning target volume. Opposing fields were used in five patients, two patients received wedged angled fields. The tumour enclosing isodose was normalised to $2 \mathrm{~Gy}$. All fields were treated Monday to Friday on a $6 \mathrm{MeV}$ Philips Linac SL6 with 2 Gy single fractions up to a median total tumour dose of 56 Gy (54-60 Gy). The following range of doses was estimated to distribute over non-tumorous brain: contralateral frontal (minimummaximum, mean (SD): 36 (19) to 46 (12) Gy; contralateral parieto-occipital: 5 (9) to 19 (16)
Gy; ipsilateral parieto-occipital: 1(2) to 13 (13) Gy. Patients visited the radiation oncologist weekly during and 6 weeks after treatment to evaluate acute side effects. Thereafter, patients were seen by the referring physician for long term follow up.

PET DATA ACQUISITION

FDG PET was performed using a 933/04-16 tomograph (CTI, Knoxville, four rings, seven planes, $8 \mathrm{~mm}$ full width at half maximum (FWHM)). The FDG was prepared as previously described. ${ }^{11}$ The head of the subjects was positioned parallel to the orbitomeatal line and was placed in an individually moulded thermoplastic head support to minimise movement during scanning. After two 10 minute transmission scans by means of a Ga-68/Ge- 68 ring source, 140 to $298 \mathrm{MBq}$ FDG were administered intravenously over 3 minutes using a constant infusion pump. In 11/19 patients, and in all healthy subjects, arterial blood samples were available for the determination of plasma radioactivity and glucose concentration. A protocol comprising one dynamic (48 minutes) and one static ( 5 minutes) emission scan was used covering the whole brain at two positions, yielding a total of 14 adjacent planes with a plane to plane distance of $8 \mathrm{~mm}$. These slices were then interpolated to 26 planes of the standard stereotactic atlas coordinate system of Talairach and Tournoux. ${ }^{12}$ Radioactivity concentrations of the resulting images were given in counts/pixel.

STATISTICAL PARAMETRIC MAPPING (SPM95)

SPM95 provides a means to investigate specific patterns of radioactivity distributions - for example, associated with brain lesions. We used SPM95 to assess the pattern of cerebral FDG uptake (as a measure of glucose metabolism) associated with a left frontal lesion (tumour) of non-irradiated and irradiated patients. For this purpose, images of patients with right sided tumours were flipped to have the lesions of all patients on the left side. Images of these patients were then compared with the images of healthy subjects and stereotactically normalised, using a series of linear and non-linear steps (SPM95). Because digitised MR images were not available, we used a standard PET template and the subjects' PET images for stereotactic normalisation. This procedure may potentially be compromised by the brain lesion itself. However, (1) no patient had a significant space occupying lesion as judged on MRI films, (2) there was no midline shift in any patient, (3) the lesion volumes were comparable in both groups, and (4) our SPM results do not consider the tumour quadrant itself. As judged by visual inspection, PET images of all patients were consistently transformed without introducing artifacts. The images were then smoothed with a gaussian filter with FWHM corresponding to the spatial resolution of the scanner. Between subject variations in global FDG uptake were removed by normalising individual global FDG uptake to an arbitrary mean by proportional scaling of the count images. By computing a $t$ statistic, images of 
Table 2 Brain areas with relative CMRGlu reductions due to left frontal tumor

\begin{tabular}{llrrrrrr}
\hline Side & Region & $X$ & $Y$ & $Z$ & Size & Decrease & $z$ Score \\
\hline Ipsilateral & Cingulate gyrus (BA 32) & -2 & 6 & 44 & 109 & $-18 \%$ & 4.52 \\
& Middle temporal gyrus (BA 39) & -50 & -64 & 24 & 253 & $-15 \%$ & 4.03 \\
Contralateral & Inferior parietal lobulus (BA 40) & 56 & -34 & 32 & 231 & $-19 \%$ & 4.14 \\
& Cingulate gyrus (BA 23) & 2 & -26 & 32 & 447 & $-13 \%$ & 3.99 \\
& Middle frontal gyrus (BA 6) & 26 & 6 & 56 & 157 & $-16 \%$ & 3.63 \\
\hline
\end{tabular}

$\mathrm{BA}=$ Brodman area; $\mathrm{X} ; \mathrm{Y} ; \mathrm{Z}=$ pixel coordinates of peak difference; size $=$ area size (number of voxels, voxel size $=2 \times 2 \times 4 \mathrm{~mm}$ ); decrease $=\%$ reduction in patients for the respective peak coordinates; z score $=$ score of peak difference (transformed $t$ value). (SPM95, patients v controls: significance level $\mathrm{p}=0.01$ ).

non-irradiated patients and normal subjects were then compared on a pixel by pixel basis. Pixels exceeding the threshold of $p=0.01$ were displayed in axial, sagittal, and coronal projections of a statistic parametric map (for methodological details see Friston et $a l^{13}$ ).

REGIONS OF INTEREST (ROI) ANALYSIS

Because SPM95 does not rely on absolute values of CMRGlu, nor does it take changes of global CMRGlu into account, absolute CMRGlu was determined in the 11 patients in whom arterial blood samples were available, and in the 12 normal subjects. For this purpose, firstly, CMRGlu (in units of $\mu \mathrm{mol} / 100 \mathrm{ml} / \mathrm{min}$ ) was calculated on the PET image sets using the original model of Sokoloff $e t a l^{14}$ with its modification for humans. ${ }^{15}$ Secondly, a standard template of elliptical ROIs was created on the stereotactically normalised PET images to compute CMRGlu profiles. The focus of this part of the study was brain contralateral rather than ipsilateral to the tumour side, because metabolism in the cortex and subcortical white matter surrounding low grade tumours can be reduced to a similar degree as in tumours themselves, which makes it difficult to exactly differentiate non-tumorous brain from tumour. In addition, contralateral brain was to a lesser amount exposed to radiotherapy than tumour itself. Thus, CMRGlu reduction in the contralateral brain of irradiated patients would strongly support the hypothesis of radiation induced side effects. The following brain regions contralateral to the tumour side were evaluated: frontomedial, frontoprefrontal, frontolateral, parietal, and occipitomedial cortex; white matter at the level of the centrum semiovale; and hemisphere contralateral to the tumour side. The ROIs were placed at the appropriate locations throughout the whole brain in an axial direction. For each subject, the resulting CMRGlu values were plotted against the relative plane offset ( $\mathrm{z}$ value, in $\mathrm{mm}$ ) related to the thalamus (thalamus $=0^{12}$ ), thus yielding individual ROI CMRGlu profiles.

Profile values for ROI CMRGlu were then averaged for each subject to result in one mean CMRGlu value per ROI per subject. In addition, ROI profiles were averaged for each
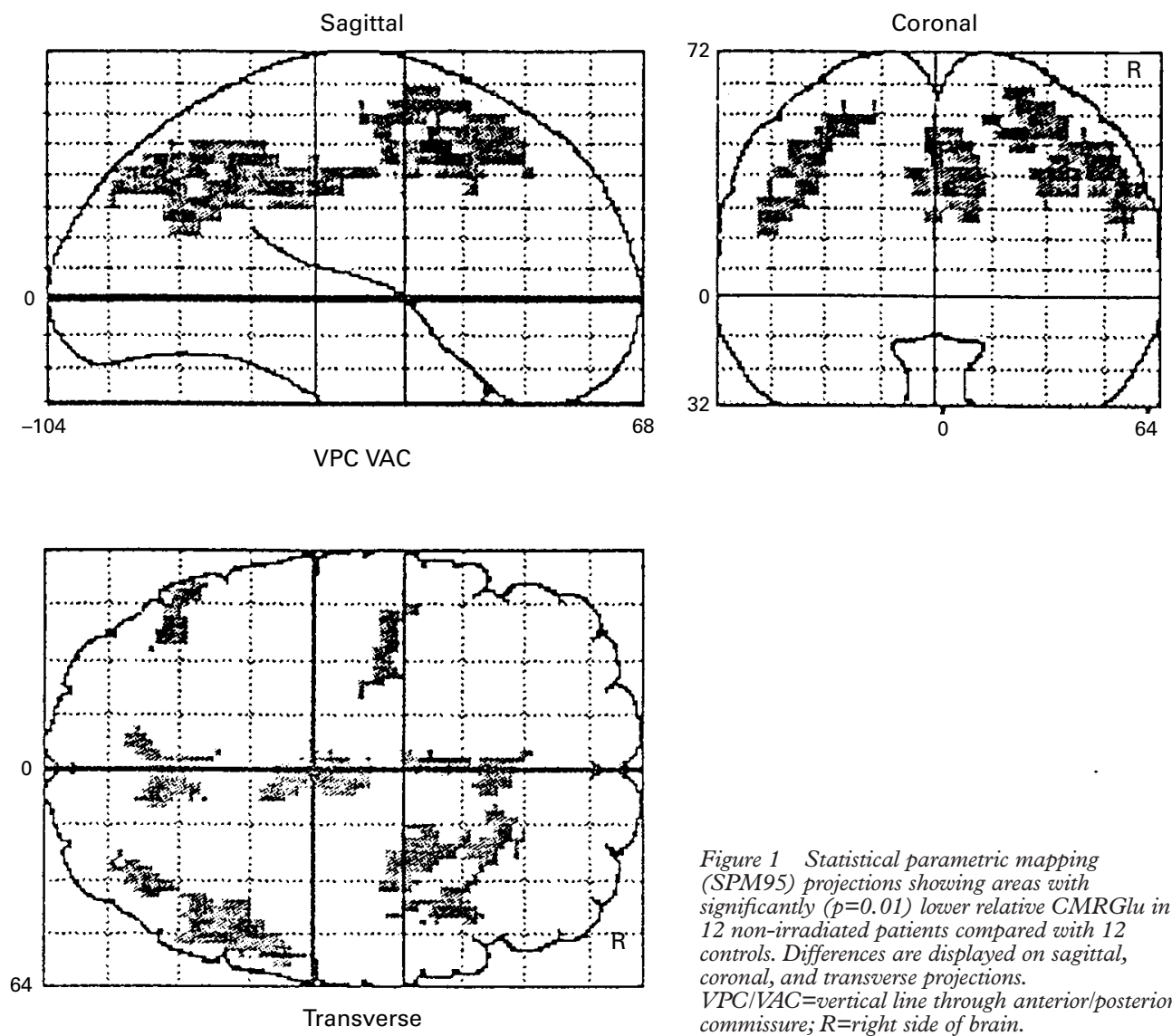

Figure 1 Statistical parametric mapping (SPM95) projections showing areas with significantly $(p=0.01)$ lower relative CMRGlu in 12 non-irradiated patients compared with 12 controls. Differences are displayed on sagittal, coronal, and transverse projections.

$V P C / V A C=$ vertical line through anterior/posterior commissure; $R=$ right side of brain. 

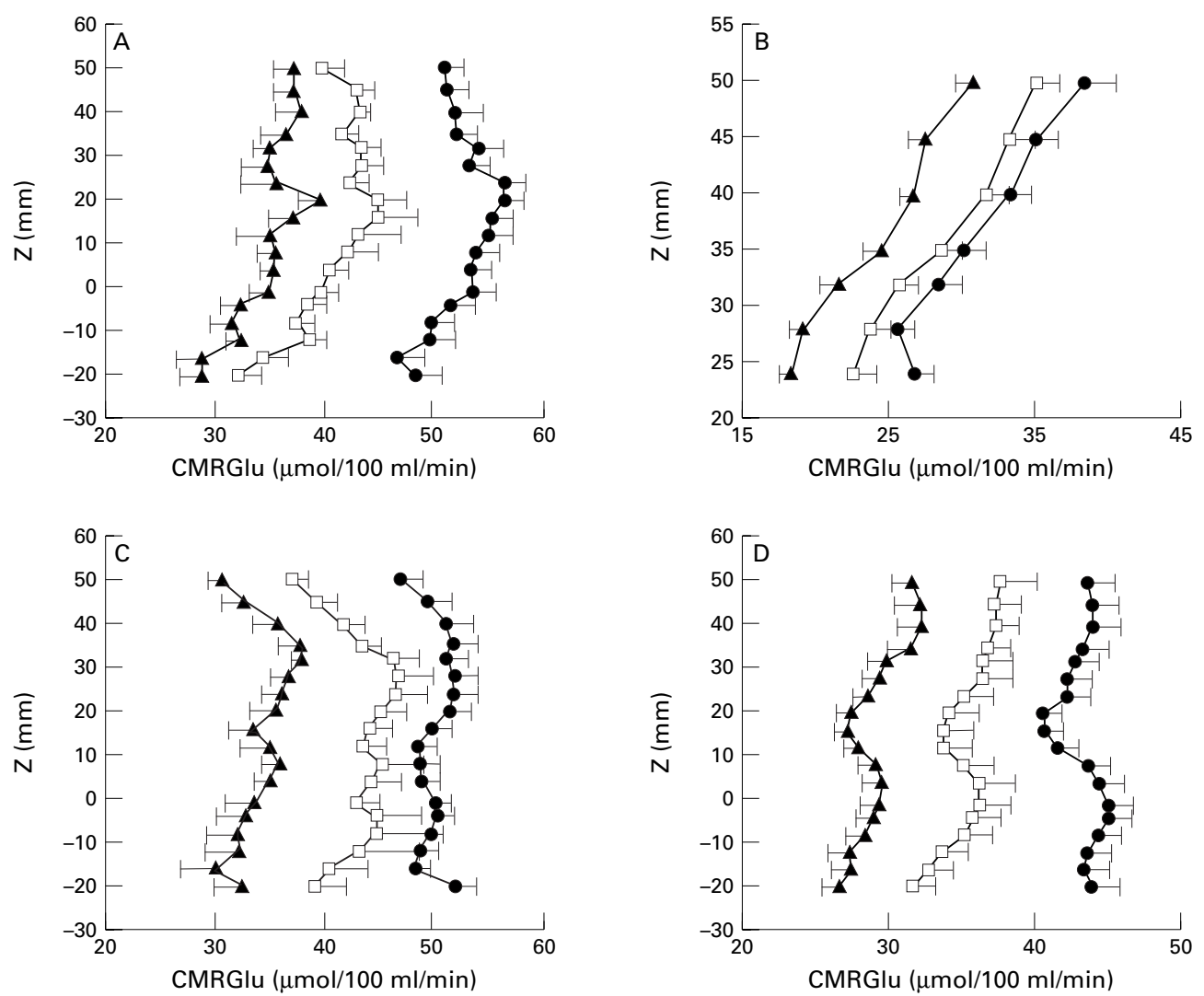

Figure $2 A-D$ Profiles of absolute CMRGlu of the contralateral hemisphere. $A=$ frontal lateral; $B=$ white matter frontal; $C=$ parietal; $D=$ hemisphere. $Z=$ offset relative to the thalamus (see Talairach and Tournoux $x^{12}$ ). Filled triangles=irradiated patients, open squares=non-irradiated patients, filled circles $=$ controls. Values are mean (SEM).

group (radiated, non-irradiated, controls) to result in one mean CMRGlu profile per location.

\section{STATISTICS}

Due to the few patients in each group, the nonparametric Kruskal-Wallis test was applied for group to group comparisons of CMRGlu ROI values. A possible relation between CMRGlu and the interval between operation with or without radiotherapy and the time of the PET study was tested using the Spearman's rank test.

\section{Results}

STATISTICAL PARAMETRIC MAPPING (SPM95)

The comparison between the 12 nonirradiated patients and 12 healthy controls disclosed a specific pattern of relatively reduced CMRGlu in patients (fig 1). This "lesion induced suppression" of CMRGlu particularly comprised the frontal brain contralateral to the tumour side, and the parietal lobe of both brain

Table 3 Absolute CMRGlu values ( $\mu \mathrm{mol} / 100 \mathrm{ml} / \mathrm{min}$ )

\begin{tabular}{llllll}
\hline ROI & RADY & & \multirow{2}{*}{ RADN } & & \multicolumn{1}{l}{ Controls } \\
\hline Frontomedial & $33.7(1.6)$ & $-14 \%$ & $39.1(1.9)$ & $-23 \%$ & $50.5(2.1)$ \\
Frontolateral & $34.5(1.7)$ & $-15 \%$ & $40.6(2.0)$ & $-22 \%$ & $52.2(1.8)$ \\
Frontoprefrontal & $34.2(1.7)$ & $-15 \%$ & $40.2(1.9)$ & $-20 \%$ & $50.0(1.9)$ \\
Parietal & $33.8(1.8)$ & $-22 \%$ & $43.1(3.1)$ & $-13 \%$ & $49.6(1.6)$ \\
Occipitomedial & $37.5(2.0)$ & $-20 \%$ & $47.0(2.9)$ & $-13 \%$ & $54.3(2.0)$ \\
White matter & $23.9(1.0)$ & $-16 \%$ & $28.5(1.5)$ & $-9 \%$ & $31.2(1.4)$ \\
Hemisphere & $28.8(1.2)$ & $-18 \%$ & $35.0(2.0)$ & $-18 \%$ & $42.9(1.5)$ \\
\hline
\end{tabular}

Values are mean (SEM). RADN=non-irradiared patients $(\mathrm{n}=7)$; RADY=irradiated patients $(n=4)$; in patients all regions refer to the brain side contralateral to the tumour. Per cent values express the CMRGlu difference between the respective groups; group differences are significant. sides. In the left frontal brain, no differences between non-irradiated patients and controls were found, which we attribute to the varying tumour locations within the frontal brain. Similar results were also obtained when irradiated patients were compared with controls (data not shown), whereas no differences in the relative CMRGlu pattern were found when non-irradiated and irradiated patients were compared. In addition, no relative increases in CMRGlu were found when patients were compared with controls. As no differences in the CMRGlu pattern were found between nonirradiated patients and controls or irradiated patients and controls, the results of the analysis as presented in table 2 are derived from the comparison between all patients $(n=19)$ and the control subjects $(n=12)$.

ROI ANALYSIS

Figure 2 A-D shows the group profiles of absolute CMRGlu values for the four irradiated and seven non-irradiated patients contralateral to the tumour, and for the 12 control subjects. For the irradiated patients, all ROIs were within the radiation field (see methods, radiotherapy). When compared with controls, the CMRGlu profiles of non-irradiated patients were shifted towards the left side (lower CMRGlu). Compared with non-irradiated patients, the profiles of irradiated patients were further shifted towards lower CMRGlu values.

The mean group CMRGlu values for each ROI are presented in table 3 and reflect the profile shifts seen in fig 2A-D. The mean 
CMRGlu reduction in non-irradiated patients versus controls averaged over all ROIs was $17 \%$, the mean difference between CMRGlu in non-irradiated patients and irradiated patients was also $17 \%$. No correlation between the time elapsed since operation with or without radiotherapy and CMRGlu was found (Spearman's rank test for all ROIs: $r<0.1, \mathrm{p}>0.1$ ).

\section{Discussion}

Neuropsychological impairment is often found in children and adults after therapeutic cranial irradiation of brain tumours. ${ }^{8}{ }^{16-20}$ Also prophylactic cranial irradiation-for example, of patients with small cell lung cancer-may cause neuropsychological disturbances. In many cases, however, it is difficult to differentiate between effects of the primary disease state and concurrent therapies. ${ }^{21}$ We used CMRGlu as a possible indicator of radiotherapy induced adverse effects in patients with operated LGAs. Our data allow the differentiation of the disease state from the radiotherapy effect. Firstly, they disclose an overall CMRGlu reduction in patients with LGAs compared with healthy subjects. This can in part be attributed to metabolic suppression of normal brain due to the presence of residual/recurrent tumour and brain damage induced by operation $\left(\right.$ diaschisis $^{10}$ ). Metabolic suppression of remote brain was evident from the SPM95 data, which showed a similar pattern for both irradiated and non-irradiated patients, and which is likely to reflect the lesion effect on remote brain glucose metabolism. Superimposed on the pattern of relative glucose metabolism, irradiated patients furthermore showed widespread reductions of absolute CMRGlu - an average of $17 \%$ compared with non-irradiated patients. Whereas lower contralateral frontal CMRGlu in irradiated patients could represent a direct consequence of radiotherapy because this brain area was also largely exposed to irradiation, that remote brain such as contralateral parietal or occipital cortex showed this reduction was unexpected. These data suggest that "localised" external beam radiotherapy applied to the treatment of frontal low grade astrocytomas may exert spatially non-confined effects.

Several neuropathological changes may underlie this CMRGlu reduction. Radiation toxicity may occur as early (within months after completion of radiotherapy) or delayed (after years) effects. Early effects are considered to correspond to transient oedema or white matter demyelination; patients may present with somnolence, mood, or memory disturbances. Delayed effects may persist and seem to be mediated by cerebrovascular changes, demyelination, and autoimmune triggered brain injury. ${ }^{22}$ Apart from frontal cortical CMRGlu reduction, CMRGlu of the contralateral white matter was also reduced in irradiated patients. Together with the CMRGlu reductions in the parietal and occipital cortex, these findings may indicate a primary damage to the white matter, which is considered the cerebral element most vulnerable to irradiation, ${ }^{23}$ and a secondary trans-synaptic suppression of adjacent or remote cortex.
Metabolic radiotherapy effects have also been shown by nuclear magnetic resonance spectroscopy. Usenius et $a l^{24}$ found a lower concentration of N-acetyl-L-aspartate (a neuron specific metabolite) in tumour adjacent brain of irradiated patients with glioma. It still needs to be determined whether these changes are primarily caused by the tumour or by the radiotherapy. Wang et $a l^{25}$ found a less suppressed metabolism of non-tumorous brain after radiotherapy of metastatic and primary brain tumours, which was most likely due to the therapeutic effect of radiotherapy on the tumour with a consequently reduced diaschisis of remote brain areas. Our results clearly discriminate between a tumour effect and a radiotherapy effect and support the view of significant remote effects of radiotherapy on brain not directly involved by the tumour.

One important factor to be considered is the time between radiotherapy and the assessment of neuropsychological functions or brain energy metabolism. Vigliani et $a l^{19}$ found attention and memory disturbances to be frequent at 6 months after radiotherapy, although these disturbances seemed to recover over subsequent years. On the contrary, Armstrong et al $l^{16}$ reported memory impairment 2 to 3 years after postoperative radiotherapy. In our series, the difference in absolute CMRGlu between both patient groups was $17 \%$ at a mean interval of 46 (irradiated patients) and 48 (non-irradiated patients) months. In addition, we found no relation between the time elapsed since operation, with or without radiotherapy, and absolute CMRGlu. Although our results are derived from only a few patients, they suggest that the radiotherapy induced effects on cerebral glucose metabolism apparently occur early after completion of radiotherapy. It is not yet clear how our metabolic findings relate to the time characteristics of the above mentioned neuropsychological reports. To clarify the exact time course of brain glucose metabolism, within subject follow up studies are required.

Our results raise the question whether decreases in CMRGlu, either regional or global, are clinically relevant, and whether they may correspond to the impaired neuropsychological performance reported in several studies of irradiated patients with low grade gliomas. ${ }^{1617} 19$ PET studies in patients with Alzheimer's disease, ${ }^{26}{ }^{27}$ brain injury, ${ }^{28}$ multiple sclerosis $^{29}$ ), and elderly healthy subjects ${ }^{30}$ showed a close relation between reductions of cerebral energy metabolism on the one hand, and the degree of subjective complaints or measurable decline in neuropsychological test performance on the other. These data thus underline the fact that measurement of cerebral energy metabolism provides a correlate of brain function. For our patient groups, it is noteworthy that CMRGlu in irradiated patients was on average $17 \%$ lower than in non-irradiated patients. In turn, CMRGlu in non-irradiated patients was to a similar magnitude lower than the mean CMRGlu of controls. This gradual decrease of CMRGlu from healthy subjects through non-irradiated 
to irradiated patients may implicate an associated decline in brain function.

This study was in part supported by the Swiss Cancer League, grant No FOR 491

1 Morantz RA. The management of the patient with a low-grade cerebral astrocytoma. In: Morantz RA, Walsh low-grade cerebral astrocytoma. In: Morantz RA, Walsh
JW, eds. Brain tumors. New York: Marcel Dekker, JW, eds. Brain

2 Touboul E, Schlienger M, Buffat L, et al. Radiation therapy with or without surgery in the management of low-grade brain astrocytomas. A retrospective study of 120 patients. Bull Cancer Radiother 1995;82:388-95.

3 Shaw EG. The low-grade glioma debate: evidence defending the position of early radiation therapy. Clin Neurosurg 1995;42:488-94.

4 Lunsford LD, Somaza S, Kondziolka D, et al. Brain astrocytomas: biopsy, then irradiation. Clin Neurosurg 1995;42:464-79.

5 Miralbell R, Balart J, Matias Guiu X, et al. Radiotherapy for supratentorial low-grade gliomas: results and prognostic factors with special focus on tumour volume parameters. Radiother Oncol 1993;27:112-6.

6 North CA, North RB, Epstein JA, et al. Low-grade cerebral astrocytomas. Survival and quality of life after radiation therapy. Cancer 1990;66:6-14.

7 Karim AB, Maat B, Hatlevoll R, et al. A randomized trial on dose-response in radiation therapy of low-grade cerebral glioma: European organization for research and treatment of cancer (EORTC) study 22844. Int 7 Radiat Oncol Biol Phys 1996;36:549-56.

8 Roman DD, Sperduto PW. Neuropsychological effects of cranial radiation: current knowledge and future directions. Int $\mathcal{F}$ Radiat Oncol Biol Phys 1995;31:983-98.

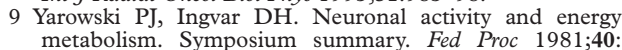
2353-62.

10 Baron JC, Bousser MG, Comar D, et al. "Crossed cerebellar diaschisis" in human supratentorial brain infarction. Ann Neurol 1980;8:128-9.

11 Hamacher K, Coenen HH, Stocklin G. Efficient stereospecific synthesis of no-carrier-added 2-[18F]-fluoro-2-deoxyD-glucose using aminopolyether supported nucleophilic substitution. F Nucl Med 1986;27:235-8.

12 Talairach J, Tournoux P. Co-planar stereotaxic atlas of the human brain. Stuttgart: Georg Thieme, 1988.

13 Friston KJ, Frith CD, Liddle PF, et al. Comparing functional (PET) images: the assessment of significant functional (PET) images: the assessment of signi
change. F Cereb Blood Flow Metab 1991;11:690-9.

14 Sokoloff L, Reivich M, Kennedy C, et al. The [14C]deoxyglucose method for the measurement of local cerebral glucose utilization: theory, procedure, and normal values in the conscious and anesthetized albino rat. $f$ Neurochem 1977;28:897-916.

15 Reivich M, Alavi A, Wolf A, et al. Glucose metabolic rate kinetic model parameter determination in humans: the lumped constants and rate constants for $[18 \mathrm{~F}]$ fluorodeoxyglucose and [11C]deoxyglucose. F Cereb Blood Flow Metab 1985;5:179-92.
16 Armstrong C, Ruffer J, Corn B, et al. Biphasic patterns of memory deficits following moderate-dose partial-brain irradiation: neuropsychologic outcome and proposed mechanisms. F Clin Oncol 1995;13:2263-71.

17 Crossen JR, Garwood D, Glatstein E, et al. Neurobehavioral sequelae of cranial irradiation in adults: a review of radiation-induced encephalopathy. F Clin Oncol 1994;12: $627-42$.

18 Taphoorn MJ, Schiphorst AK, Snoek FJ, et al. Cognitive functions and quality of life in patients with low-grade gliomas: the impact of radiotherapy. Ann Neurol 1994;36: 48-54.

19 Vigliani MC, Sichez N, Poisson M, et al. A prospective study of cognitive functions following conventional radiotherapy for supratentorial gliomas in young adults: 4-year results. Int 7 Radiat Oncol Biol Phys 1996;35:527-33.

20 Chadderton RD, West CG, Schulz S, et al. Radiotherapy in the treatment of low-grade astrocytomas. II. The physical and cognitive sequelae. Childs Nerv Syst 1995;11:443-8.

21 Van Oosterhout AG, Ganzevles PG, Wilmink JT, et al. Sequelae in long-term survivors of small cell lung cancer. Int F Radiat Oncol Biol Phys 1996;34:1037-44.

22 Fajardo LF. Pathology of radiation injury. New York: Masson Publishing, USA, 1982.

23 Corn BW, Yousem DM, Scott CB, et al. White matter changes are correlated significantly with radiation dose. Observations from a randomized dose-escalation trial for malignant glioma (Radiation Therapy Oncology Group 83-02). Cancer 1994;74:2828-35.

24 Usenius $\mathrm{T}$, Usenius JP, Tenhunen $\mathrm{M}$, et al. Radiationinduced changes in human brain metabolites as studied by $1 \mathrm{H}$ nuclear magnetic resonance spectroscopy in vivo. Int $\mathfrak{F}$ Radiat Oncol Biol Phys 1995;33:719-24.

25 Wang GJ, Volkow ND, Lau YH, et al. Glucose metabolic changes in nontumoral brain tissue of patients with brain tumor following radiotherapy: a preliminary study. $\mathcal{f} \mathrm{Com}-$ put Assist Tomogr 1996;20:709-14.

26 Sultzer DL, Mahler ME, Mandelkern MA, et al. The relationship between psychiatric symptoms and regional cortical metabolism in Alzheimer's disease. F Neuropsychiatry Clin Neurosci 1995;7:476-84.

27 Nyback H, Nyman H, Blomqvist G, et al. Brain metabolism in Alzheimer's dementia: studies of $11 \mathrm{C}$-deoxyglucose accumulation, CSF monoamine metabolites and neuropsychological test performance in patients and healthy subjects. F Neurol Neurosurg Psychiatry 1991;54:672-8.

28 Gross H, Kling A, Henry G, et al. Local cerebral glucose metabolism in patients with long-term behavioral and cognitive deficits following mild traumatic brain injury. $\mathcal{F} \mathrm{Neu}$ ropsychiatry Clin Neurosci 1996;8:324-34.

29 Brooks DJ, Leenders KL, Head G, et al. Studies on regional cerebral oxygen utilization and cognitive function in multiple sclerosis. F Neurol Neurosurg Psychiatry 1984;47:118291.

30 Small GW, Okonek A, Mandelkern MA, et al. Ageassociated memory loss: initial neuropsychological and cerebral metabolic findings of a longitudinal study. Int Psychogeriatr 1994;6:23-44. 